\title{
Novel One-Step Nucleic Acid Amplification (OSNA) Application in Prostate Cancer. What Can We Learn from its Usage in Other Cancer Entities? A Systematic Review
}

\author{
Mercè Cuadras \\ Vall d'Hebron University Hospital \\ Jacques Planas ( $\sim$ jplanas@vhebron.net) \\ Vall d'Hebron University Hospital \\ Ana Celma \\ Vall d'Hebron University Hospital \\ Lucas Regis \\ Vall d'Hebron University Hospital \\ Inés M. de Torres \\ Vall d'Hebron University Hospital \\ M. Eugenia Semidey \\ Vall d'Hebron University Hospital \\ Enrique Trilla \\ Vall d'Hebron University Hospital \\ Juan Morote \\ Vall d'Hebron University Hospital
}

\section{Research Article}

Keywords: One-Step Nucleic Acid Amplification, OSNA, Cytokeratin 19, CK19, prostate cancer, lymph node metastases

Posted Date: September 16th, 2021

DOl: https://doi.org/10.21203/rs.3.rs-840288/v1

License: (c) (1) This work is licensed under a Creative Commons Attribution 4.0 International License. Read Full License 


\section{Abstract}

Background: Lymph node ( $L N)$ status is a key prognostic factor in the decision-making process of prostate cancer (PCa) management. Sectioning and haematoxylin and eosin (H\&E) staining technique remain the gold standard for the evaluation of LN metastases despite some limitations, especially low sensitivity in detecting an accurate tumour burden within the LN, as well as a subjective and time-consuming result. One-step nucleic acid amplification (OSNA) quantifies mRNA copies of cytokeratin 19 (CK19) in a fast, objective, automated, and reproducible way, raising a general interest to explore its utility for lymphatic metastasis identification in different malignancies.

Methods: To present the latest evidence related to the detection of LN metastases in several tumours by using OSNA compared with the conventional H\&E method, a systematic review of articles published since March 2021 was conducted using PubMed, Cochrane Library, and Web of Science databases. References from primary papers and review articles were checked to obtain further potential studies. Our procedure for evaluating records identified during the literature search followed the Preferred Reporting Items for Systematic Reviews and Meta-analyses criteria.

Results: Twenty five studies were included. LN from six different groups of tumours: breast, gastrointestinal, gynecological, lung, head and neck and prostate cancers has been assessed. OSNA was compared with post-operative formalin-fixed paraffin-embedded tissue sections with H\&E staining as the reference standard. Contingency tables were created, and concordance rate, sensitivity, specificity and predictive values were reported. Seventeen studies analysed the discordant cases using different techniques.

Conclusion: OSNA method has a high diagnostic accuracy for the detection of LN metastases in several CK19 expressing tumours. Available evidence encourages its usage in PCa patients to improve LN staging and prognosis.

\section{Background}

Prostate cancer $(\mathrm{PCa})$ is the second most incident neoplasm and the fifth cancer specific cause of male mortality worldwide [1]. Upon diagnosis, PCa is classified into major risk categories based on TNM clinical stage, biopsy Gleason score, and serum prostate specific antigen (PSA) levels. High-risk patients associate more biochemical recurrence, metastatic progression, and PCa related death [2].

Pelvic lymph nodes (LN) represent the most common site of metastases in PCa patients considered for surgical treatment. According to the series reviewed, the risk of LN invasion at radical prostatectomy ranges between 3 and $24 \%$, and could be even higher in high-risk PCa patients [3].

Conventional imaging techniques, such as computed tomography and magnetic resonance imaging, have low sensitivity for the detection of LN metastases [4]. The introduction of positron emission tomography with different radiotracers such as 11C-Choline and especially 68Ga-PSMA has increased the sensitivity to detect LN metastases. The 68Ga-PSMA has demonstrated $>90 \%$ specificity with sensitivity rates of $33-99 \%$ depending on serum PSA [5]. As $\leq 5 \mathrm{~mm}$ metastases are mostly missed by these techniques [6], extended pelvic lymph node dissection (ePLND) remains the most accurate staging procedure despite the fact that up to $20 \%$ of patients will present some kind of complication after its performance [7].

Due to the limited sensitivity of imaging techniques in the detection of small metastases, different nomograms based on preoperative characteristics have been described in order to define which PCa patient will truly benefit from an ePLND [8,9].

Lymphadenectomy extent and histological nodal evaluation have an impact on the staging and consequent prognosis of the disease. The gold-standard procedure consists of a macroscopic identification of the LN, followed by its sectioning into 3-4 $\mathrm{mm}$ slices, and then analysis through haematoxylin and eosin (H\&E) staining of at least one slice per LN [10]. Main limitations of this approach are metastatic tissue allocation and interobserver bias, as well as being costly and timeconsuming. 
New methods, such as serial section analysis (slices with a thickness of 1-2 mm), immunohistochemistry (IHC), and molecular tissue analysis using Reverse Transcription-Polymerase Chain Reaction (RT-PCR) for PSA have demonstrated a higher sensitivity to identifying low tumour burden in the nodes [11]. High costs, the time required for the analysis, and some limitations to standardization have hindered their routine application, though they remain relevant in clinical research.

In 2008, an innovative biomolecular technique called One-Step Nucleic Acid Amplification (OSNA) was introduced in Europe to assess LN metastases. OSNA is an automated system based on reverse transcription loop-mediated isothermal amplification method, able to quantify copies of cytokeratin 19 (CK19) mRNA. CK19 is a marker expressed by several solid tumours of epithelial origin, but not by healthy lymphatic tissue [12]. OSNA allows a quick and accurate analysis of the tumour burden of entire LN tissue in an objective, automated, and reproducible way [13-15]. It has been proven useful in different cancer entities, such as breast, colorectal, gastric, endometrial, cervical, lung, and head and neck cancer, achieving a high sensitivity and specificity in the detection of LN involvement, as well as a high concordance compared to comprehensive histopathological examination, in some cases even comparable to ultra-staging [16].

OSNA was first applied in the intraoperative analysis of sentinel lymph node (SLN) in breast cancer, introducing an objective evaluation of the nodal tissue, as well as reducing the required time and effort by the laboratory personnel. More than 10 years ago, Tsujimoto et al. [15] demonstrated the correlation between OSNA and conventional histopathological analysis of the SLN in breast cancer and defined the cut-off values for the distinction between macrometastases, micrometastases, and unaffected tissue. Since then, more than 200 studies have been published and the application range of OSNA was extended to other cancer entities [17].

The available scientific and clinical evidence, together with the mentioned characteristics, has introduced OSNA in current national and European clinical guidelines as an alternative technique for the determination of lymphatic involvement in breast cancer through SLN analysis [18]. Moreover, data available from studies in colorectal cancer demonstrated that OSNA is a valid technique for the detection of lymphatic involvement also in this cancer entity [19]. Hence, OSNA is now included in the recommendations for the determination of biomarkers in colorectal carcinoma [20].

Interestingly, the quantitative outcome of the OSNA assay was identified as useful tool to predict, during surgery, non-SLN involvement in breast and gynecological cancer, thus supporting tailoring of surgical procedure [21]. In breast and colorectal cancer, OSNA was shown to provide also prognostic information [22].

Regarding urological tumours, based on previous studies that demonstrated the expression of CK19 in PCa tissue, Winter et al. showed that the OSNA method can detect CK19 mRNA in 100\% of primary PCa tumours regardless of Gleason score and even more effectively than CK19 IHC expression, suggesting the valid application of this technique in LN evaluation [23]. In a very recent study, Engels et al. [24] demonstrated that OSNA can identify nodal metastases at an equivalent or, in cases of micrometastases, better rate than enhanced histological examination in PCa patients, confirming its promising use in intraoperative decision-making in personalized LN surgery.

To set up future clinical use of OSNA in PCa, the aim of this review is to analyse the available evidence of this technique in different tumours and propose short-term course of actions to translate the validated concepts and successes from the other malignancies to PCa.

\section{Methods}

\section{Search strategy}

To retrieve all relevant papers published before the end of March 2021, three databases including PubMed, Cochrane Library, and Web of Science were searched by two independent reviewers combining the following Medical Subject Headings: onestep nucleic acid amplification, OSNA, lymph nodes, lymph node metastases, cytokeratin 19, CK19. References from primary papers and review articles were checked to obtain further potential studies. Our procedure for evaluating records identified 
during the literature search followed the Preferred Reporting Items for Systematic Reviews and Meta-analyses (PRISMA) criteria [25]. Disagreements were resolved through discussion.

\section{Eligible criteria}

We defined study eligibility using the PICO strategy (patient population, intervention, comparison, and outcomes) [26]. A study was considered relevant to this review according to the following criteria: 1) Adult patients with confirmed cancer, eligible for surgical treatment and undergoing SLN biopsy (SLNB) or regional lymphadenectomy; 2) patients did not undergo any neoadjuvant treatment; 3 ) the main objective was to compare OSNA using fresh LN with postoperative standard formalinfixed paraffin-embedded (FFPE)-H\&E analysis; 4) LN were dissected and analysed using both OSNA and the standard technique at the same time; 5) the pathological examination method was fully described; 6 ) results were reported per node (minimum 100 nodes); 7) sufficient data was available to calculate true-positive, false-positive, false-negative and truenegative values. We limited these criteria to English original studies. Review articles, meta-analysis, conference abstracts, and letters were excluded.

\section{Study selection}

The flow diagram of study selection process was outlined in Fig. 1. A total of 244 potentially relevant studies were identified using the searching terms described. 89 duplicated studies were initially excluded. After screening titles and abstracts, 102 papers were removed. From the remaining 52 studies, 28 were excluded after full text review because the comparison was made with intraoperative frozen section or touch imprint cytology as a reference method, less than 100 nodes were included, analysis was performed per patient, or insufficient data was available to form $2 \times 2$ tables.

Finally, 25 studies met all the requirements to be considered in the systematic review.

\section{Quality assessment}

Quality Assessment of Diagnostic Accuracy Studies 2 (QUADAS-2) was used as an evidence-based quality assessment tool [27]. QUADAS-2 comprised four domains: patient selection, index test, reference standard, and flow and timing. The risk of bias of each study was evaluated by two independent reviewers as low "+", high "-" or unclear "?" risk.

The QUADAS-2 results summarized in Table 1 suggest a low risk of bias and a moderate to high overall quality of all 25 included studies.

Table 1. Risk of bias of included studies 
a)

\begin{tabular}{|c|c|c|c|c|c|c|c|c|}
\hline \multirow[b]{3}{*}{ Group } & \multirow[b]{3}{*}{ Author } & \multicolumn{7}{|c|}{ Risk of bias } \\
\hline & & \multicolumn{2}{|c|}{ Patient selection } & \multicolumn{2}{|c|}{ Index test } & \multicolumn{2}{|c|}{ Reference Standard } & \multirow{2}{*}{$\begin{array}{l}\text { Flow and timing } \\
\text { Were all samples } \\
\text { included? }\end{array}$} \\
\hline & & $\begin{array}{c}\text { Selection } \\
\text { criteria } \\
\text { clearly } \\
\text { described? }\end{array}$ & $\begin{array}{c}\text { Clinical data } \\
\text { available? }\end{array}$ & $\begin{array}{c}\text { Threshold } \\
\text { pre- } \\
\text { specified? }\end{array}$ & $\begin{array}{l}\text { Uninterpretable } \\
\text { results } \\
\text { analysed? }\end{array}$ & $\begin{array}{c}\text { Pathology } \\
\text { method } \\
\text { description? }\end{array}$ & $\begin{array}{c}\text { Blinded } \\
\text { pathologist? }\end{array}$ & \\
\hline \multirow{8}{*}{ 1) } & Tsujimoto et al. [15] & + & + & + & + & + & ? & + \\
\hline & Feldman et al. [28] & + & + & + & + & + & + & + \\
\hline & Le Frère-Belda et al. [29] & + & + & + & ? & + & + & - \\
\hline & Vegué et al. [30] & + & + & + & - & + & ? & - \\
\hline & Wang et al. [31] & + & + & + & - & + & + & + \\
\hline & Pathmanathan et al. [32] & + & + & + & + & + & $?$ & + \\
\hline & Banerjee et al. [33] & + & + & + & + & + & ? & + \\
\hline & Li et al. [34] & + & + & + & + & + & + & - \\
\hline \multirow{7}{*}{ 2) } & Croner et al. [35] & + & + & + & + & + & $?$ & - \\
\hline & Yamamoto et al. [36] & + & + & + & + & + & + & - \\
\hline & Guller et al. [37] & + & + & + & + & + & ? & + \\
\hline & Yamamoto et al. [38] & + & + & + & + & + & $?$ & - \\
\hline & Yaguchi et al. [39] & $?$ & + & + & + & ? & + & + \\
\hline & Kumagai et al[40](28) & + & + & + & + & + & + & - \\
\hline & Shimada et al. [41] & $?$ & + & + & $?$ & + & $?$ & - \\
\hline \multirow{4}{*}{ 3) } & Okamoto et al. $[42](29)$ & + & + & + & + & + & ? & + \\
\hline & Nagai et al. [43] & - & $?$ & + & + & + & ? & ? \\
\hline & Fanfani et al. [44] & + & + & + & $?$ & + & $?$ & + \\
\hline & Kost'un et al. [45](30) & + & + & + & $?$ & + & $?$ & + \\
\hline \multirow{3}{*}{ 4) } & Inoue et al. [46] & $?$ & + & + & $?$ & + & $?$ & + \\
\hline & Nakagawa et al. [47] & + & + & + & + & + & + & - \\
\hline & Escalante-Pérez et al. [48] & + & + & + & + & + & + & + \\
\hline \multirow{2}{*}{ 5) } & Goda et al. [49] & $?$ & $?$ & + & $?$ & + & $?$ & + \\
\hline & Sofia del Carmen et al. [50] & + & $?$ & + & $?$ & + & ? & - \\
\hline 6) & Engels et al. [24] & + & $?$ & + & ? & + & ? & - \\
\hline
\end{tabular}

b)

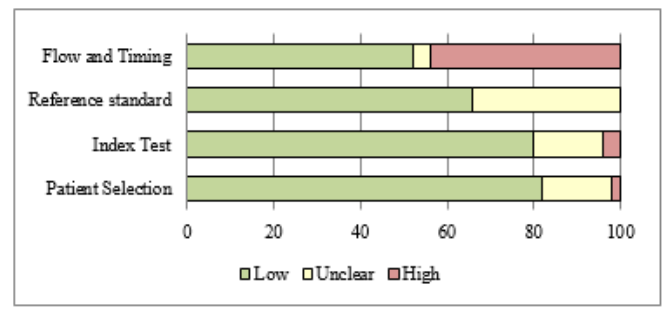

a) Assessment of risk of bias. Summary of risk of bias for each study; +: low risk of bias; -: high risk of bias; ?: unclear risk of bias.

b) Risk of bias graph about each risk of bias item presented as percentages across all included studies

\section{Results}

The 25 eligible studies have been published between January 2007 and March 2021. Our review included SLN and non-SLN from six different groups of tumours: 1) breast [15,28-34], 2) gastrointestinal -colorectal [35-38] and gastric cancers [3941]-, 3) gynecological -cervical [42] and endometrial cancers [43-45]-, 4) lung [46-48], 5) head and neck -head and neck squamous cell carcinomas (HNSCC) [49] and thyroid cancers [50]- and 6) PCa [24]. All studies were prospectively designed. OSNA was considered as index test and a threshold of 250 copies of CK19 mRNA per $\mu \mathrm{L}$ was fixed to differentiate between negative $(<250$ copies $/ \mu \mathrm{L})$ and positive $(\geq 250$ copies $/ \mu \mathrm{L})$ results. OSNA was compared with post-operative FFPE tissue 
sections with H\&E staining as the reference standard. 11 studies also included also CK19 IHC analysis in addition to H\&E staining and OSNA. A LN was cut into at least two parts (depending on LN size) and divided between OSNA assay and pathology. Contingency tables were created, and concordance rate was reported. 17 studies analysed the discordant cases (OSNA + / H\&E -; OSNA - / H\&E+) using different techniques.

Detailed characteristics are shown in Table 2.

Sensitivity, specificity, positive predictive value (PPV), negative predictive value (NPV), and concordance are listed in Table 3. Discordant cases are included in the reported results.

Table 2. Characteristics of included studies 


\begin{tabular}{|c|c|c|c|c|c|c|c|c|c|}
\hline Group & Author & Year & Country & $\begin{array}{l}\text { Tumour } \\
\text { type }\end{array}$ & $\begin{array}{l}\text { No. } \\
\text { Patients }\end{array}$ & $\begin{array}{l}\text { No. } \\
\text { Nodes }\end{array}$ & $\begin{array}{l}\text { Reference } \\
\text { method }\end{array}$ & $\begin{array}{l}\text { CK19 } \\
\text { IHC }\end{array}$ & $\begin{array}{l}\text { Analysis } \\
\text { of } \\
\text { discordant } \\
\text { cases }\end{array}$ \\
\hline \multirow[t]{8}{*}{ 1) } & $\begin{array}{l}\text { Tsujimoto et } \\
\text { al. [15] }\end{array}$ & 2007 & Japan & Breast & 101 & 325 & $\begin{array}{l}\text { H\&E and } \\
\mathrm{IHC}\end{array}$ & yes & yes \\
\hline & $\begin{array}{l}\text { Feldman et al. } \\
\text { [28] }\end{array}$ & 2011 & USA & Breast & 496 & 1044 & $\begin{array}{l}\text { H\&E and } \\
\mathrm{IHC}\end{array}$ & yes & yes \\
\hline & $\begin{array}{l}\text { Le Frère-Belda } \\
\text { et al. [29] }\end{array}$ & 2012 & France & Breast & 233 & 503 & $\begin{array}{l}\text { H\&E and } \\
\text { IHC }\end{array}$ & yes & yes \\
\hline & $\begin{array}{l}\text { Vegué et al. } \\
\text { [30] }\end{array}$ & 2012 & Spain & Breast & 57 & 567 & $H \& E$ & yes & no \\
\hline & $\begin{array}{l}\text { Wang et al. } \\
\text { [31] }\end{array}$ & 2012 & China & Breast & 552 & 1188 & H\&E & no & yes \\
\hline & $\begin{array}{l}\text { Pathmanathan } \\
\text { et al. [32] }\end{array}$ & 2014 & Australia & Breast & 98 & 170 & $\begin{array}{l}\text { H\&E and } \\
\text { IHC }\end{array}$ & no & no \\
\hline & $\begin{array}{l}\text { Banerjee et al. } \\
\text { [33] }\end{array}$ & 2014 & UK & Breast & 170 & 268 & $\mathrm{H} \& \mathrm{E}$ & no & no \\
\hline & Li et al. [34] & 2015 & China & Breast & 115 & 311 & $H \& E$ & no & yes \\
\hline \multirow[t]{7}{*}{ 2) } & $\begin{array}{l}\text { Croner et al. } \\
\text { [35] }\end{array}$ & 2010 & Germany & Colorectal & 184 & 184 & $\begin{array}{l}\text { H\&E and } \\
\mathrm{IHC}\end{array}$ & yes & yes \\
\hline & $\begin{array}{l}\text { Yamamoto et } \\
\text { al. [36] }\end{array}$ & 2011 & Japan & Colorectal & 85 & 385 & $\mathrm{H} \& \mathrm{E}$ & no & yes \\
\hline & $\begin{array}{l}\text { Guller et al. } \\
\text { [37] }\end{array}$ & 2012 & Switzerland & Colorectal & 22 & 313 & $\begin{array}{l}\text { H\&E and } \\
\text { IHC }\end{array}$ & yes & yes \\
\hline & $\begin{array}{l}\text { Yamamoto et } \\
\text { al. [38] }\end{array}$ & 2016 & Japan & Colorectal & 204 & 1925 & $\mathrm{H} \& \mathrm{E}$ & no & no \\
\hline & $\begin{array}{l}\text { Yaguchi et al. } \\
\text { [39] }\end{array}$ & 2011 & Japan & Gastric & 32 & 162 & $H \& E$ & no & yes \\
\hline & $\begin{array}{l}\text { Kumagai et al. } \\
\text { [40] }\end{array}$ & 2014 & Japan & Gastric & 61 & 394 & H\&E & no & yes \\
\hline & $\begin{array}{l}\text { Shimada et al. } \\
\text { [41] }\end{array}$ & 2019 & Japan & Gastric & 43 & 439 & H\&E & no & yes \\
\hline \multirow[t]{4}{*}{ 3) } & $\begin{array}{l}\text { Okamoto et al. } \\
{[42](29)}\end{array}$ & 2013 & Japan & Cervical & 32 & 130 & H\&E & no & yes \\
\hline & $\begin{array}{l}\text { Nagai et } \\
\text { al. [43] }\end{array}$ & 2015 & Japan & Endometrial & 35 & 137 & H\&E & no & yes \\
\hline & $\begin{array}{l}\text { Fanfani et al. } \\
\text { [44] }\end{array}$ & 2018 & Italy & Endometrial & 40 & 110 & $\begin{array}{l}\text { H\&E and } \\
\text { IHC }\end{array}$ & yes & no \\
\hline & $\begin{array}{l}\text { Kost'un et al. } \\
{[45](30)}\end{array}$ & 2019 & $\begin{array}{l}\text { Czech } \\
\text { Republic }\end{array}$ & Endometrial & 58 & 135 & $\begin{array}{l}\text { H\&E and } \\
\text { IHC }\end{array}$ & yes & no \\
\hline \multirow[t]{3}{*}{ 4) } & $\begin{array}{l}\text { Inoue et al. } \\
\text { [46] }\end{array}$ & 2012 & Japan & Lung & 49 & 165 & $\begin{array}{l}\text { H\&E and } \\
\text { IHC }\end{array}$ & no & no \\
\hline & $\begin{array}{l}\text { Nakagawa et } \\
\text { al. [47] }\end{array}$ & 2016 & Japan & Lung & 111 & 410 & $\mathrm{H} \& \mathrm{E}$ & no & yes \\
\hline & $\begin{array}{l}\text { Escalante- } \\
\text { Pérez et al. } \\
\text { [48] }\end{array}$ & 2019 & Spain & Lung & 160 & 705 & $\begin{array}{l}\mathrm{H} \& \mathrm{E} \text { and } \\
\mathrm{IHC}\end{array}$ & yes & no \\
\hline
\end{tabular}




\begin{tabular}{|c|c|c|c|c|c|c|c|c|c|}
\hline \multirow[t]{2}{*}{ 5) } & Goda et al. [49] & 2012 & Japan & HNSCC & 65 & 312 & $H \& E$ & no & yes \\
\hline & $\begin{array}{l}\text { Sofía del } \\
\text { Carmen et al. } \\
\text { [50] }\end{array}$ & 2016 & Spain & Thyroid & 37 & 267 & $\begin{array}{l}\text { H\&E and } \\
\mathrm{IHC}\end{array}$ & yes & yes \\
\hline 6) & $\begin{array}{l}\text { Engels et al. } \\
{[24]}\end{array}$ & 2021 & Germany & Prostate & 64 & 534 & $\begin{array}{l}\text { H\&E and } \\
\text { IHC }\end{array}$ & yes & yes \\
\hline
\end{tabular}

CK19: Cytokeratin 19; FN: False negative; FP: False positive; HNSCC: Head and neck squamous cell carcinoma; H\&E: Hematoxylin and eosin; IHC: Immunohistochemistry; No: Number of; TN: True negative; TP: True positive.

Table 3. OSNA accuracy compared with histopathological examination in included tumours. 


\begin{tabular}{|c|c|c|c|c|c|c|c|c|}
\hline \multirow[t]{2}{*}{ Group } & \multirow[t]{2}{*}{ Author } & \multicolumn{2}{|c|}{ H\&E positive } & \multicolumn{2}{|c|}{ H\&E negative } & \multirow[t]{2}{*}{ Sensitivity } & \multirow[t]{2}{*}{ Specificity } & \multirow[t]{2}{*}{ Concordance } \\
\hline & & $\begin{array}{l}\text { OSNA } \\
\text { negative }\end{array}$ & $\begin{array}{l}\text { OSNA } \\
\text { positive }\end{array}$ & $\begin{array}{l}\text { OSNA } \\
\text { negative }\end{array}$ & $\begin{array}{l}\text { OSNA } \\
\text { positive }\end{array}$ & & & \\
\hline \multirow[t]{8}{*}{ 1) } & $\begin{array}{l}\text { Tsujimoto et al. } \\
\text { [15] }\end{array}$ & $0.6(2)$ & $\begin{array}{l}13.2 \\
(43)\end{array}$ & $85(276)$ & $1.2(4)$ & 95.6 & 98.6 & $\begin{array}{l}98.2 \\
(319 / 325)\end{array}$ \\
\hline & $\begin{array}{l}\text { Feldman et al. } \\
\text { [28] }\end{array}$ & $3(31)$ & $\begin{array}{l}10.2 \\
(107)\end{array}$ & $\begin{array}{l}83.1 \\
(868)\end{array}$ & $3.6(38)$ & 77.5 & 95.8 & $\begin{array}{l}93.4 \\
(975 / 1044)\end{array}$ \\
\hline & $\begin{array}{l}\text { Le Frère-Belda et } \\
\text { al. [29] }\end{array}$ & $2.4(12)$ & $\begin{array}{l}10.1 \\
(51)\end{array}$ & $\begin{array}{l}82.1 \\
(413)\end{array}$ & $5.4(27)$ & 80.9 & 93.9 & $\begin{array}{l}92.2 \\
(464 / 503)\end{array}$ \\
\hline & Vegué et al. [30] & $0(0)$ & $1.1(6)$ & $\begin{array}{l}92.1 \\
(522)\end{array}$ & $6.9(39)$ & 100 & 93 & $\begin{array}{l}93.1 \\
(528 / 567)\end{array}$ \\
\hline & Wang et al. [31] & $2.6(31)$ & $\begin{array}{l}13.4 \\
(159)\end{array}$ & 78 (927) & $6(71)$ & 83.7 & 92.9 & $\begin{array}{l}91.4 \\
(1086 / 1188)\end{array}$ \\
\hline & $\begin{array}{l}\text { Pathmanathan } \\
\text { et al. [32] }\end{array}$ & $1.8(3)$ & $\begin{array}{l}14.7 \\
(25)\end{array}$ & $\begin{array}{l}80.6 \\
(137)\end{array}$ & $3(5)$ & 89.3 & 96.5 & $\begin{array}{l}95.3 \\
(162 / 170)\end{array}$ \\
\hline & $\begin{array}{l}\text { Banerjee et al. } \\
\text { [33] }\end{array}$ & $0.7(2)$ & $\begin{array}{l}14.6 \\
(39)\end{array}$ & 81 (217) & $3.7(10)$ & 95.1 & 95.6 & $\begin{array}{l}95.5 \\
(256 / 268)\end{array}$ \\
\hline & Li et al. [34] & $2(6)$ & $9.6(30)$ & $\begin{array}{l}85.5 \\
(266)\end{array}$ & $2.9(9)$ & 83.3 & 96.7 & $\begin{array}{l}95.2 \\
(296 / 311)\end{array}$ \\
\hline \multirow[t]{7}{*}{ 2) } & Croner et al. [35] & $1.6(3)$ & $\begin{array}{l}20.1 \\
(37)\end{array}$ & $\begin{array}{l}75.5 \\
(139)\end{array}$ & $2.7(5)$ & 92.5 & 96.5 & $\begin{array}{l}95.7 \\
(176 / 184)\end{array}$ \\
\hline & $\begin{array}{l}\text { Yamamoto et al. } \\
\text { [36] }\end{array}$ & $1(4)$ & $\begin{array}{l}20.5 \\
(79)\end{array}$ & $\begin{array}{l}76.6 \\
(295)\end{array}$ & $1.8(7)$ & 95.2 & 97.7 & $\begin{array}{l}97.1 \\
(374 / 385)\end{array}$ \\
\hline & Guller et al. [37] & $0.6(2)$ & $\begin{array}{l}16.3 \\
(51)\end{array}$ & $\begin{array}{l}79.6 \\
(249)\end{array}$ & $3.5(11)$ & 96.2 & 95.7 & $\begin{array}{l}95.8 \\
(300 / 313)\end{array}$ \\
\hline & $\begin{array}{l}\text { Yamamoto et al. } \\
\text { [38] }\end{array}$ & $1(20)$ & $\begin{array}{l}6.5 \\
(125)\end{array}$ & $\begin{array}{l}89.2 \\
(1717)\end{array}$ & $3.3(63)$ & 86.2 & 96.5 & $\begin{array}{l}95.7 \\
(1842 / 1925)\end{array}$ \\
\hline & $\begin{array}{l}\text { Yaguchi et al. } \\
\text { [39] }\end{array}$ & $3.1(5)$ & $\begin{array}{l}24.7 \\
(40)\end{array}$ & $\begin{array}{l}69.8 \\
(113)\end{array}$ & $2.5(4)$ & 88.9 & 96.6 & $\begin{array}{l}94.4 \\
(153 / 162)\end{array}$ \\
\hline & $\begin{array}{l}\text { Kumagai et al. } \\
\text { [40] }\end{array}$ & $2.3(9)$ & $\begin{array}{l}11.4 \\
(45)\end{array}$ & $\begin{array}{l}82.7 \\
(326)\end{array}$ & $3.6(14)$ & 83.3 & 95.9 & $\begin{array}{l}94.2 \\
(371 / 394)\end{array}$ \\
\hline & $\begin{array}{l}\text { Shimada et al. } \\
{[41]}\end{array}$ & $1.8(8)$ & $3.2(14)$ & $\begin{array}{l}93.9 \\
(412)\end{array}$ & $1.1(5)$ & 63.6 & 98.8 & $\begin{array}{l}97 \\
(426 / 439)\end{array}$ \\
\hline \multirow[t]{4}{*}{ 3) } & $\begin{array}{l}\text { Okamoto et al. } \\
\text { [42] }\end{array}$ & $2.3(3)$ & $2.3(3)$ & $\begin{array}{l}93.8 \\
(122)\end{array}$ & $1.5(2)$ & 50 & 98.4 & $\begin{array}{l}96.2 \\
(125 / 130)\end{array}$ \\
\hline & Nagai et al. [43] & $2.2(3)$ & $\begin{array}{l}10.2 \\
(14)\end{array}$ & $\begin{array}{l}86.9 \\
(119)\end{array}$ & $0.7(1)$ & 82.4 & 99.2 & $\begin{array}{l}97.1 \\
(133 / 137)\end{array}$ \\
\hline & $\begin{array}{l}\text { Fanfani et al. } \\
\text { [44] }\end{array}$ & $0.9(1)$ & $7.2(8)$ & $86.4(95)$ & $5.4(6)$ & 88.9 & 94.1 & $\begin{array}{l}93.6 \\
(103 / 110)\end{array}$ \\
\hline & $\begin{array}{l}\text { Kost'un et al. } \\
\text { [45] }\end{array}$ & $0.7(1)$ & $7.4(10)$ & $\begin{array}{l}78.5 \\
(106)\end{array}$ & $\begin{array}{l}13.3 \\
(18)\end{array}$ & 90.9 & 85.5 & $\begin{array}{l}85.9 \\
(116 / 135)\end{array}$ \\
\hline \multirow[t]{3}{*}{ 4) } & Inoue et al. [46] & $0.6(1)$ & $\begin{array}{l}11.5 \\
(19)\end{array}$ & $\begin{array}{l}87.3 \\
(144)\end{array}$ & $0.6(1)$ & 95 & 99.3 & $\begin{array}{l}98.8 \\
(163 / 165)\end{array}$ \\
\hline & $\begin{array}{l}\text { Nakagawa et al. } \\
\text { [47] }\end{array}$ & $2.9(12)$ & $\begin{array}{l}11.5 \\
(47)\end{array}$ & $\begin{array}{l}81.2 \\
(333)\end{array}$ & $4.4(18)$ & 79.7 & 94.5 & $\begin{array}{l}92.7 \\
(380 / 410)\end{array}$ \\
\hline & Escalante-Pérez & $0.1(1)$ & $4.8(34)$ & 91.3 & 3.7 (26) & 97.1 & 96.1 & 96.2 \\
\hline
\end{tabular}


et al. [48]

5)

Goda et al. [49]

$2.6(8)$

$5.2(14)$

Sofía del

Carmen et al.

[50]

$\begin{array}{lll}6) \quad \text { Engels et al. [24] } & 2.2(12) & 14.2 \\ & & \end{array}$

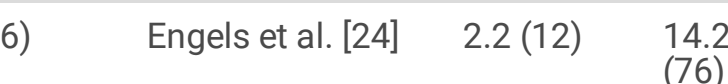

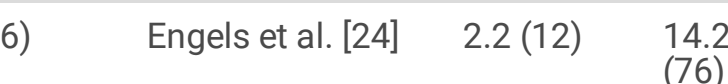

(644)

$17(53) \quad 77.2$

(241)

28.1

(75)

60 (160)

$6.7(18)$

$3.2(10) \quad 86.9$

86.9

96

$(678 / 705)$

94.2

(294/312)

88

$(235 / 267)$

(76)

80.5

(452)

$3.4(18)$

91.4

100

98.8

$(528 / 534)$

Figures are expressed as percentages and (number of cases) in parentheses

H\&E: Hematoxylin and eosin; No: Number of; OSNA: One-step nucleic acid amplification.

\section{Discussion}

LN status is a key prognostic factor in the decision-making process of cancer management. For a long time, sectioning and H\&E staining technique has been the gold standard for the evaluation of LN metastases. Even though it remains an adequate tool, some limitations have been described, especially low sensitivity in detecting the accurate tumour burden, mainly as a consequence of sampling bias [10], as well as a subjective and time-consuming result. To overcome these limitations, OSNA assay has been developed as a fast, objective, automated, and reproducible way to examine the whole LN, raising a general interest to explore its utility for lymphatic metastases identification in different tumours.

OSNA gives a quantitative result of CK19 mRNA copies, which is present in several simple epithelia but is not expressed in healthy lymphatic tissue [12]. CK19 was initially proposed as a marker for the detection of LN metastases in breast cancer, where it is found in up to $98 \%$ of cases [51]. In 2007, Tsujimoto et al. [15] determined $250 \mathrm{copies} / \mu$ l as the optimum cut-off point to define a positive axillary LN in breast cancer population. Nonetheless, it is known that the number of positive LN and the size of metastases are significant prognostic factors in most tumours. Therefore, it was also established a second cut-off point of 5,000 copies/ $\mu$ lo distinguish between micro and macrometastases [15]. Subsequent studies have confirmed these values and all the results reflected in this review are based on them.

In 2013 V. Peg et al. [21] defined the concept of total tumour load (TTL) as the total CK19 mRNA copies of all positive SLNs. TTL serves as a predictive and prognosis value, providing more accurate staging than pathological findings. Accordingly, different OSNA studies in breast SLN have set cut-off values in order to predict the axillary LN status; some of which (10,000-15,000 copies) are already included in clinical guidelines [52, 53]. In 2017, Rakislova et al. [54] explored its utility to predict recurrences in colorectal carcinoma, and a recent study confirmed that a TTL $\geq 6,000 \mathrm{copies} / \mu \mathrm{l}$ was associated with worse disease free survival in those patients [55].

The analysis of SLN in breast cancer patients is still its main clinical application, but over the years OSNA has raised interest of the pathology community for a more accurate LN staging in other cancer entities. In the last decade, several reports comparing OSNA with histopathological examination have been published, but after a systematic review of the available literature, to date only two studies related to PCa have been found $[23,24]$.

All the articles included in this review compare OSNA assay with postoperative H\&E staining in the same LN. There is a general concordance between OSNA and standard H\&E of over $85 \%$. No full information about discordant cases is available, but we have found not only different explanations for them but also heterogeneity in its analysis. Main justifications for the discordant cases are tumour allocation bias (TAB), and more rarely low or no tumour CK19 expression and contamination by other epithelial cells [46].

As CK19 is the single molecular marker used in OSNA assay, low tumour CK19 expression may result in a false-negative OSNA case. Different CK19 expression levels have been described for other malignancies such as colorectal (94.1\%) [36],

Page $10 / 17$ 
gastric (98.6\%) [39], gynecological (98\%) [43], lung (96\%) [48], HNSCC (91.1\%) [49] or PCa (100\%) [23]. Moreover, certain tumour subtypes are more likely to the lack of CK19 expression, as observed in metaplastic and lobular breast carcinomas [56]. Interestingly, Goda et al. [49] performed a CK19 IHC in primary HNSCC as a first step when analyzing discordant results, detecting no expression of CK19 in 75\% of those cases. Aiming to reduce false-negative cases in breast, thyroid and lung carcinomas, Vegué et al. [30], del Carmen et al. [50], and Escalante Pérez et al. [48] verified the presence of CK19 in primary tumour by IHC before LN analysis. For a more accurate interpretation of the results, we encourage future researchers to include CK19 expression in primary tumours as patient inclusion criteria.

One of the main advantages of OSNA is its capacity to analyse the whole LN, leading to a more precise diagnosis than conventional histology. However, to properly compare both techniques on the same node, it is mandatory to split it, leading to a possible misdetection of metastasis by one of the methods, which is called TAB. Trying to justify the discrepancies by the need for sectioning, 17 studies have reported different strategies: second-round OSNA analysis, CK19 IHC, exhaustion of the paraffin-embedded SLN slices, CK19 Western blot, or CK19 qRT-PCR. Most of the metastases from the discrepancies were confirmed thanks to these strategies.

Regarding PCa, CK19 has been found not only in neoplastic tissue but also in basal and luminal cells of normal, dysplastic and benign hyperplastic tissues, although complete data concerning CK19-RNA levels is still missing [57]. In 2018, Winter et al. [23] published the first OSNA assay in PCa. A total of 20 primary PCa tumours from intermediate-high risk PCa patients (Gleason $\geq 7$ ) were analysed. A central slice was analysed by OSNA, while surrounding slices were sent for both conventional H\&E staining and CK19 IHC. PCa was confirmed by H\&E in all 20 samples; OSNA was able to detect CK19 mRNA in 100\% of cases, ranging from 320 to 250.000 copies/ $\mu$ l while IHC did not detect CK19 in one specimen. Given the small sample size and the high tumour burden of the selected patients, we cannot fully extrapolate these findings for all PCa patients.

Recently, Engels et al. [24] published a second OSNA assay in PCa, including a total of 574 SLNs from 64 PCa patients. SLNs were assessed by conventional H\&E staining and OSNA assay. The comparison between both techniques showed a sensitivity, specificity and concordance of $84.2 \%, 96.1 \%$ and $94.4 \%$, respectively.

To date, the ePLND remains the most accurate staging procedure [58], but individual assessment of all dissected LNs is laborious and time consuming. Using OSNA, LNs can be pooled together and analysed in a few samples as already done in colorectal cancer [54]. In fact, Engels et al. [24] suggested that such approach could be feasible to analysed PCa LNs as a faster and economic alternative. Moreover, it could be interesting to define a TTL value to predict the risk of early biochemical recurrence and the need of adjuvant treatment.

When compared to histopathological examination, OSNA offers the advantage to obtain objective and quantitative data about tumour load of the whole LN in a fast and effortless way, avoiding interobserver variability. Multicentric prospective studies with large cohorts of patients may investigate the the benefits of OSNA and its impact as a potential prognostic factor for PCa. In contrast, a potential disadvantage of examining the whole LN with OSNA is that there is no tissue left for subsequent histopathological examination following complete homogenization. Nonetheless, RNA-based molecular tests are possible using OSNA lysate, thus allowing any follow-up molecular testing. Moreover, fresh lymphatic tissue requires a thorough dissection to avoid missing nodes, which must be completely separated from fat tissue by a trained pathologist. It is also important to note that in the case of a coexisting neoplasm, OSNA cannot define which primary tumour the metastases come from and is unable to distinguish LN tumour cells from other benign epithelial inclusions. Therefore, it is imperative to exclude other cancers with the same lymphatic drainage and avoid contaminations.

Despite these concerns, the results reported show a high specificity, concordance rate, and NPV of OSNA assay when compared with the H\&E method. Specifically, in breast cancer patients, that high NPV provides enough evidence to become the gold standard for SLN evaluation. As slicing is required for the analysis per node, a concordance of $100 \%$ cannot be achieved due to TAB. A high concordance rate of over $85 \%$ suggests that OSNA could be an alternative technique to 
histopathological examination in terms of its ability to detect lymph node metastases. Future investigations may bring light to the clinical impact of OSNA in PCa as well as to its potential predictive and prognostic roles.

\section{Conclusion}

OSNA is a suitable tool to standardize LN evaluation in most CK19 expressing tumours due to the possibility to analyse the whole LN in a fast, objective, automated, and reproducible way. OSNA assay has demonstrated a high diagnostic accuracy for the detection of LN tumour burden also in PCa, but more studies are needed to confirm its validation. Moreover, intraoperative use of OSNA may be a great opportunity to set up sentinel-guided LN dissection in PCa.

\section{Abbreviations}

CK19: Cytokeratin 19; ePLND: Extended pelvic lymph node dissection; FFPE: Formalin-fixed paraffin-embedded; HNSCC: Head and neck squamous cell carcinomas; H\&E: Haematoxylin and eosin; IHC: Immunohistochemistry; LN: Lymph node; OSNA: One-step nucleic acid amplification; PCa: Prostate cancer; PSA: Prostate specific antigen; PRISMA: Preferred reporting items for systematic reviews and meta-analyses; QUADAS 2: Quality assessment of diagnostic accuracy studies 2; RT-PCR: Reverse transcription-polymerase chain reaction; SLN: Sentinel lymph node; TAB: Tumour allocation bias; TTL: Total tumour load.

\section{Declarations}

\section{Ethics approval and consent to participate}

Not applicable.

\section{Consent for publication}

Not applicable.

\section{Availability of data and material}

All the data and materials analysed are included in the main paper. Further data are available from the corresponding author on reasonable request.

\section{Competing interests}

The authors declare that they have no competing interests.

\section{Funding}

The authors would like to thank SYSMEX for their insightful suggestions and careful reading of the manuscript. Furthermore authors want to thank the funding support for the English adaptation and the Open Access fee.

\section{Authors' contributions}

JM participated in the evidence acquisition. JM, ET, and JP contributed to writing the manuscript. All authors read and approved the final manuscript.

\section{Acknowledgements}

Not applicable.

\section{References}


1. Bray F, Ferlay J, Soerjomataram I, Siegel RL, Torre LA, Jemal A. Global cancer statistics 2018: Globocan estimates of incidence and mortality worldwide for 36 cancers in 185 countries. CA Cancer J Clin. 2018 Nov;68(6):394-424.

2. Bianchi L, Gandaglia G, Fossati N, Suardi N, Moschini M, Cucchiara V, et al. Pelvic Lymph Node Dissection in Prostate Cancer: Indications, Extent and Tailored Approaches. Urol J. 2017 Jan;84(1):9-19.

3. Joniau S, Briganti A, Gontero P, Gandaglia G, Tosco L, Fieuws S, et al. Stratification of High-risk Prostate Cancer into Prognostic Categories: A European Multi-institutional Study. Eur Urol. 2015 Jan;67(1):157-64.

4. Hövels AM, Heesakkers RAM, Adang EM, Jager GJ, Strum S, Hoogeveen YL, et al. The diagnostic accuracy of CT and MRI in the staging of pelvic lymph nodes in patients with prostate cancer: a meta-analysis. Clin Radiol. 2008 Apr;63(4):387-95.

5. Corfield J, Perera M, Bolton D, Lawrentschuk N. 68Ga-prostate specific membrane antigen (PSMA) positron emission tomography (PET) for primary staging of high-risk prostate cancer: a systematic review. World J Urol. 2018 Apr;36(4):519-27.

6. van Leeuwen PJ, Emmett L, Ho B, Delprado W, Ting F, Nguyen Q, et al. Prospective evaluation of 68Gallium-prostatespecific membrane antigen positron emission tomography/computed tomography for preoperative lymph node staging in prostate cancer. BJU Int. 2017 Feb;119(2):209-15.

7. Briganti A, Chun FK-H, Salonia A, Suardi N, Gallina A, Da Pozzo LF, et al. Complications and Other Surgical Outcomes Associated with Extended Pelvic Lymphadenectomy in Men with Localized Prostate Cancer. Eur Urol. 2006 Nov;50(5):1006-13.

8. Briganti A, Larcher A, Abdollah F, Capitanio U, Gallina A, Suardi N, et al. Updated nomogram predicting lymph node invasion in patients with prostate cancer undergoing extended pelvic lymph node dissection: the essential importance of percentage of positive cores. Eur Urol. 2012 Mar;61(3):480-7.

9. Gandaglia G, Ploussard G, Valerio M, Mattei A, Fiori C, Fossati N, et al. A Novel Nomogram to Identify Candidates for Extended Pelvic Lymph Node Dissection Among Patients with Clinically Localized Prostate Cancer Diagnosed with Magnetic Resonance Imaging-targeted and Systematic Biopsies. Eur Urol. 2019 Mar;75(3):506-14.

10. Lawrence WD, Association of Directors of Anatomic and Surgical Pathology. ADASP recommendations for processing and reporting of lymph node specimens submitted for evaluation of metastatic disease. Virchows Arch. 2001 Nov;439(5):601-3.

11. Schostak M, Krause H, Miller K, Schrader M, Kempkensteffen C, Kollermann J. Does the molecular staging in pelvic lymph nodes improve the detection of relevant prostate cancer metastases? An assessment after 6years. BJU Int. 2007 Jun;99(6):1409-14.

12. Moll R, Franke WW, Schiller DL, Geiger B, Krepler R. The catalog of human cytokeratins: Patterns of expression in normal epithelia, tumours and cultured cells. Cell. 1982 Nov;31(1):11-24.

13. Nagamine K, Hase T, Notomi T. Accelerated reaction by loop-mediated isothermal amplification using loop primers. Mol Cell Probes. 2002 Jun;16(3):223-9.

14. Notomi T, Okayama H, Masubuchi H, Yonekawa T, Watanabe K, Amino N, et al. Loop-mediated isothermal amplification of DNA. Vol. 28, Nucleic Acids Research. 2000.

15. Tsujimoto M, Nakabayashi K, Yoshidome K, Kaneko T, Iwase T, Akiyama F, et al. One-step Nucleic Acid Amplification for Intraoperative Detection of Lymph Node Metastasis in Breast Cancer Patients. Clin Cancer Res. 2007 Aug;13(16):480716.

16. Fanfani F, Monterossi G, Di Meo ML, La Fera E, Dell'orto F, Gioè A, et al. Standard ultra-staging compared to one-step nucleic acid amplification for the detection of sentinel lymph node metastasis in endometrial cancer patients: A retrospective cohort comparison. Int J Gynecol Cancer. 2020 Mar;30(3):372-7.

17. Tranoulis A, Georgiou D, Yap J, Attard-Montalto S, Twigg J, Elattar A, et al. The evolving role of one-step nucleic acid amplification (OSNA) for the intra-operative detection of lymph node metastases: A diagnostic accuracy meta-analysis. European Journal of Surgical Oncology. W.B. Saunders Ltd; 2020. 
18. Perry N, Broeders M, de Wolf C, Törnberg S, Holland R, von Karsa L. European guidelines for quality assurance in breast cancer screening and diagnosis. Fourth edition - Summary document. Vol. 19, Annals of Oncology. Ann Oncol; 2008. p.614-22.

19. Hiyoshi Y, Akiyoshi T, Fukunaga Y. The advantage of one-step nucleic acid amplification for the diagnosis of lymph node metastasis in colorectal cancer patients. Vol. 5, Annals of Gastroenterological Surgery. Blackwell Publishing Ltd; 2021. p.60-6.

20. García-Alfonso P, García-Carbonero R, García-Foncillas J, Pérez-Segura P, Salazar R, Vera R, et al. Update of the recommendations for the determination of biomarkers in colorectal carcinoma: National Consensus of the Spanish Society of Medical Oncology and the Spanish Society of Pathology. Clin Transl Oncol. 2020 Nov;22(11):1976-91.

21. Peg V, Espinosa-Bravo M, Vieites B, Vilardell F, Antúnez JR, De Salas MS, et al. Intraoperative molecular analysis of total tumour load in sentinel lymph node: A new predictor of axillary status in early breast cancer patients. Breast Cancer Res Treat. 2013 May;139(1):87-93.

22. Peg V, Sansano I, Vieites B, Bernet L, Cano R, Córdoba A, et al. Role of total tumour load of sentinel lymph node on survival in early breast cancer patients. Breast. 2017 Jun;33:8-13.

23. Winter A, Engels S, Goos P, Süykers M-C, Henke R-P, Gerullis H, et al. Detection of CK19 mRNA Using One-step Nucleic Acid Amplification (OSNA) in Prostate Cancer: Preliminary Results. J Cancer. 2018;9(24):4611-7.

24. Engels S, Brautmeier L, Reinhardt L, Wasylow C, Hasselmann F, Henke RP, et al. Evaluation of fast molecular detection of lymph node metastases in prostate cancer patients using one-step nucleic acid amplification (Osna). Cancers (Basel). 2021 Mar;13(5):1-12.

25. Shamseer L, Moher D, Clarke M, Ghersi D, Liberati A, Petticrew M, et al. Preferred reporting items for systematic review and meta-analysis protocols (PRISMA-P) 2015: elaboration and explanation. BMJ. 2015 Jan;350:g7647.

26. Huang X, Lin J, Demner-Fushman D. Evaluation of PICO as a knowledge representation for clinical questions. AMIA . Annu Symp Proc. 2006;359-63.

27. Whiting PF, Rutjes AWS, Westwood ME, Mallett S, Deeks JJ, Reitsma JB, et al. QUADAS-2: A Revised Tool for the Quality Assessment of Diagnostic Accuracy Studies. Ann Intern Med. 2011 Oct;155(8):529.

28. Feldman S, Krishnamurthy S, Gillanders W, Gittleman M, Beitsch PD, Young PR, et al. A Novel Automated Assay for the Rapid Identification of Metastatic Breast Carcinoma in Sentinel Lymph Nodes. Cancer. 2011;117(12):2599-607.

29. Le Frère-Belda M-A, Bats A-S, Gillaizeau F, Poulet B, Clough KB, Nos C, et al. Diagnostic performance of one-step nucleic acid amplification for intraoperative sentinel node metastasis detection in breast cancer patients. Int J Cancer. 2012 May;130(10):2377-86.

30. Vegué LB, Rojo F, Hardisson D, Iturriagagoitia AC, Panadés MJ, Velasco A, et al. Comparison of molecular analysis and histopathology for axillary lymph node staging in primary breast cancer: results of the B-CLOSER-I study. Diagn Mol Pathol. 2012 Jun;21(2):69-76.

31. Wang Y, Ou-yang T, Wu J, Liu Y, Cao X, Sun X, et al. Comparative study of one-step nucleic acid amplification assay, frozen section, and touch imprint cytology for intraoperative assessment of breast sentinel lymph node in Chinese patients. Cancer Sci. 2012 Nov;103(11):1989-93.

32. Pathmanathan N, Renthawa J, French JR, Edstrom-Elder E, Hall G, Mahajan H, et al. Intraoperative sentinel lymph node assessment in breast cancer: a comparison of rapid diagnostic method based on CK19 mRNA expression and imprint cytology. ANZ J Surg. 2014 Oct;84(10):730-4.

33. Banerjee SM, Michalopoulos N V, Williams NR, Davidson T, El Sheikh S, McDermott N, et al. Detailed evaluation of one step nucleic acid (OSNA) molecular assay for intra-operative diagnosis of sentinel lymph node metastasis and prediction of non-sentinel nodal involvement: Experience from a London Teaching Hospital. Breast. 2014 Aug;23(4):378-84.

34. Li D, Xu X, Chen J, Chen J, Yang B, Yang W, et al. Utility of one-step nucleic acid amplification (OSNA) assay in detecting breast cancer metastases of sentinel lymph nodes in a Chinese population. Breast Cancer. 2015 Mar;22(2):135-40. 
35. Croner RS, Schellerer V, Demund H, Schildberg C, Papadopulos T, Naschberger E, et al. One Step Nucleic Acid Amplification (OSNA) - a new method for lymph node staging in colorectal carcinomas. J Transl Med. 2010 Sep;8:83.

36. Yamamoto H, Sekimoto M, Oya M, Yamamoto N, Konishi F, Sasaki J, et al. OSNA-Based Novel Molecular Testing for Lymph Node Metastases in Colorectal Cancer Patients: Results from a Multicenter Clinical Performance Study in Japan. Ann Surg Oncol. 2011 Jul;18(7):1891-8.

37. Güller U, Zettl A, Worni M, Langer I, Cabalzar-Wondberg D, Viehl CT, et al. Molecular investigation of lymph nodes in colon cancer patients using one-step nucleic acid amplification (OSNA): a new road to better staging? Cancer. 2012 Dec;118(24):6039-45.

38. Yamamoto H, Tomita N, Inomata M, Furuhata T, Miyake Y, Noura S, et al. OSNA-Assisted Molecular Staging in Colorectal Cancer: A Prospective Multicenter Trial in Japan. Ann Surg Oncol. 2016 Feb;23(2):391-6.

39. Yaguchi Y, Sugasawa H, Tsujimoto H, Takata H, Nakabayashi K, Ichikura T, et al. One-Step Nucleic Acid Amplification (OSNA) for the Application of Sentinel Node Concept in Gastric Cancer. Ann Surg Oncol. 2011 Aug;18(8):2289-96.

40. Kumagai K, Yamamoto N, Miyashiro I, Tomita Y, Katai H, Kushima R, et al. Multicenter study evaluating the clinical performance of the OSNA assay for the molecular detection of lymph node metastases in gastric cancer patients. Gastric Cancer. 2014 Apr;17(2):273-80.

41. Shimada A, Takeuchi H, Nishi T, Mayanagi S, Fukuda K, Suda K, et al. Utility of the one-step nucleic acid amplification assay in sentinel node mapping for early gastric cancer patients. Gastric Cancer. 2019 Oct.

42. Okamoto S, Niikura H, Nakabayashi K, Hiyama K, Matoda M, Takeshima N, et al. Detection of sentinel lymph node metastases in cervical cancer: assessment of KRT19 mRNA in the one-step nucleic acid amplification (OSNA) method. Gynecol Oncol. 2013 Sep;130(3):530-6.

43. Nagai T, Niikura H, Okamoto S, Nakabayashi K, Matoda M, Utsunomiya H, et al. A New Diagnostic Method for Rapid Detection of Lymph Node Metastases Using a One-Step Nucleic Acid Amplification (OSNA) Assay in Endometrial Cancer. Ann Surg Oncol. 2015 Mar;22(3):980-6.

44. Fanfani F, Monterossi G, Ghizzoni V, Rossi ED, Dinoi G, Inzani F, et al. One-Step Nucleic Acid Amplification (OSNA): A fast molecular test based on CK19 mRNA concentration for assessment of lymph-nodes metastases in early stage endometrial cancer. PLoS One. 2018; 13(4):e0195877.

45. Kostun J, Pešta M, Sláma J, Slunéčko R, Vlasák P, Bouda J, et al. One-step nucleic acid amplification vs ultrastaging in the detection of sentinel lymph node metastasis in endometrial cancer patients. J Surg Oncol. 2019 Mar; 119(3):361-9.

46. Inoue M, Hiyama K, Nakabayashi K, Morii E, Minami M, Sawabata N, et al. An accurate and rapid detection of lymph node metastasis in non-small cell lung cancer patients based on one-step nucleic acid amplification assay. Lung Cancer. 2012 Dec; 78(3):212-8.

47. Nakagawa $K$, Asamura $H$, Tsuta $K$, Nagai $K$, Yamada E, Ishii G, et al. The novel one-step nucleic acid amplification (OSNA) assay for the diagnosis of lymph node metastasis in patients with non-small cell lung cancer (NSCLC): Results of a multicenter prospective study. Lung Cancer. 2016 Jul; 97:1-7.

48. Escalante Pérez M, Hermida Romero MT, Otero Alén B, Álvarez Martínez M, Fernández Prado R, de la Torre Bravos M, et al. Detection of lymph node metastasis in lung cancer patients using a one-step nucleic acid amplification assay: a single-centre prospective study. J Transl Med. 2019 Dec; 17(1):233.

49. Goda H, Nakashiro K, Oka R, Tanaka H, Wakisaka H, Hato N, et al. One-step nucleic acid amplification for detecting lymph node metastasis of head and neck squamous cell carcinoma. Oral Oncol. 2012 Oct; 48(10):958-63.

50. del Carmen S, Gatius S, Franch-Arcas G, Baena JA, Gonzalez O, Zafon C, et al. Concordance study between one-step nucleic acid amplification and morphologic techniques to detect lymph node metastasis in papillary carcinoma of the thyroid. Hum Pathol. 2016 Feb; 48:132-41.

51. Chu PG, Weiss LM. Keratin expression in human tissues and neoplasms. Histopathology. 2002 May; 40(5):403-39.

52. Bernet L, Piñero A, Vidal-Sicart S, Peg V, Giménez J, Algara M, et al. Consenso sobre la biopsia selectiva del ganglio centinela en el cáncer de mama. Revisión 2013 de la Sociedad Española de Senología y Patología Mamaria. Rev Esp 
Patol. 2014.

53. Oncoguía SEGO: Cáncer infiltrante de mama. Guías de práctica clínica en cáncer ginecológico y mamario. Publicaciones SEGO, Junio 2017.

54. Rakislova N, Montironi C, Aldecoa I, Fernandez E, Bombi JA, Jimeno M, et al. Lymph node pooling: a feasible and efficient method of lymph node molecular staging in colorectal carcinoma. J Transl Med. 2017; 15(1):14.

55. Archilla I, Díaz-Mercedes S, Aguirre JJ, Tarragona J, Machado I, Rodrigo MT, et al. Lymph Node Tumour Burden Correlates With Tumour Budding and Poorly Differentiated Clusters: A New Prognostic Factor in Colorectal Carcinoma? Clin Transl Gastroenterol. 2021 Mar; 12(3):e00303.

56. Bettington M, Lakhani SR, Ung OA. Is the one-step nucleic acid amplification assay better for intra-operative assessment of breast sentinel nodes? ANZ J Surg. 2014 Oct; 84(10):725-9.

57. Peehl DM, Sellers RG, McNeal JE. Keratin 19 in the adult human prostate: tissue and cell culture studies. Cell Tissue Res. 1996 Jul; 285(1):171-6.

58. Mottet N, Van Den Bergh RCN, Briers E, Van Den Broeck T, Cumberbatch MG, De Santis M, et al. EAU-EANM-ESTRO-ESURSIOG Guidelines on Prostate Cancer-2020 Update. Part 1: Screening, Diagnosis, and Local Treatment with Curative Intent. 2020.

\section{Figures}


Potentially relevant studies from PubMed (155), Cochrane Library (15) and ScienceDirect (74) one-step nucleic acid amplification, OSNA, lymph nodes, lymph node metastases, cytokeratin 19, CK19 Filters: clinical research literature, full text availability, english language, humans
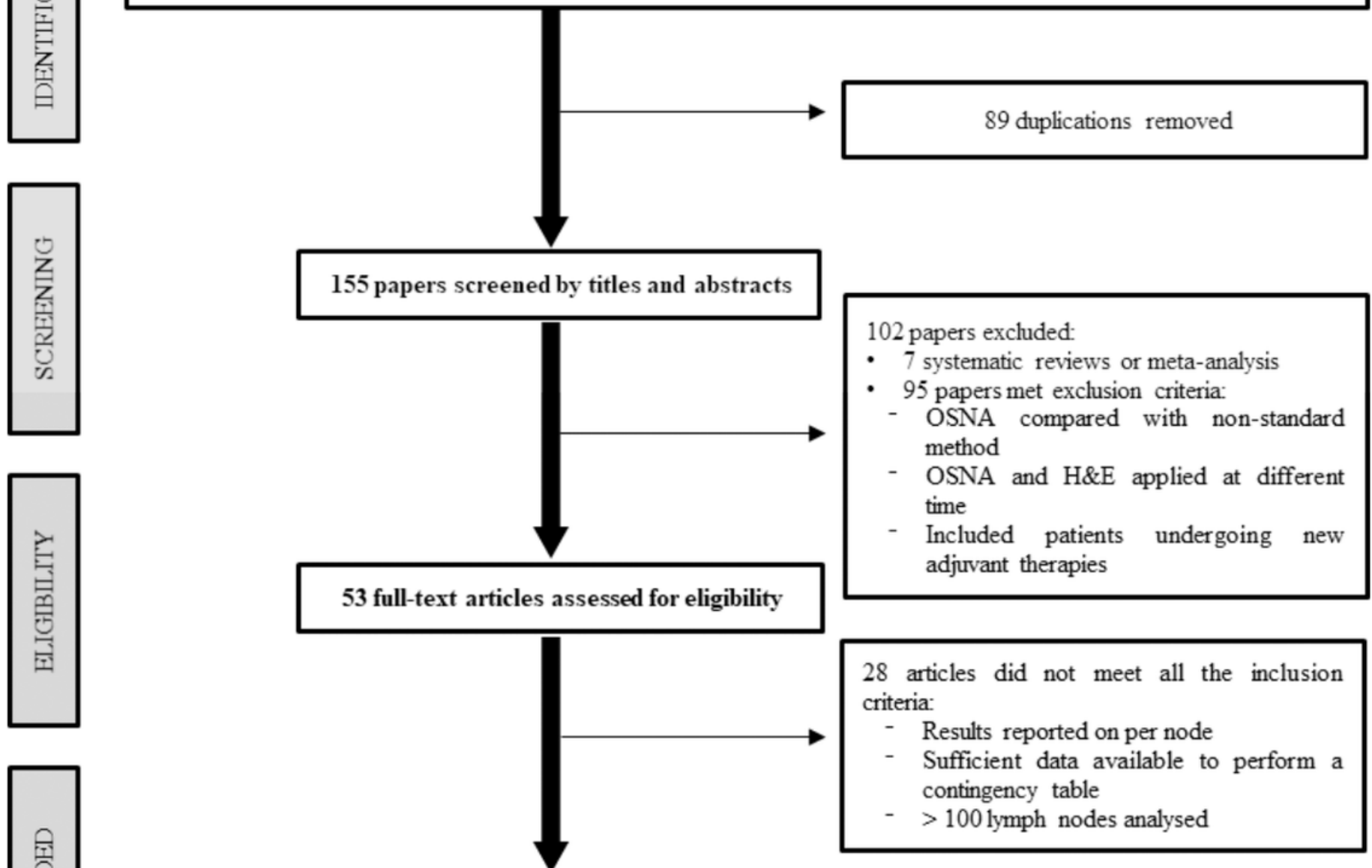

25 studies included after review of full text

Figure 1

The flow diagram of study selection process 